\title{
The effects of clinical simulation on stress, anxiety, and hemodynamic parameters among the candidates for coronary angiography
}

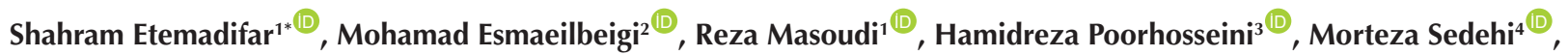 \\ Zahra Hadian Jazi ${ }^{(\mathbb{D}}$ \\ ${ }^{1}$ Community-Oriented Nursing Midwifery Research Center, Department of Adult and elderly Nursing, Shahrekord University of Medical \\ Sciences, Shahrekord, Iran \\ ${ }^{2}$ Master of Nursing, Department of Nursing, School of Nursing \& Midwifery, Shahrekord University of Medical Sciences, Shahrekord, Iran \\ ${ }^{3}$ Tehran Heart Center, North Kargar Avenue, Tehran, Iran \\ ${ }^{4}$ Department of Statistics and Epidemiology, School of Public Health, Shahrekord University of MedicalSciences, Shahrekord, Iran \\ ${ }^{5}$ Department of Medical Surgical, Faculty of Nursing and Mideifery, Isfahan University of Medical Sciences, Isfahan, Iran
}

\begin{abstract}
Background and aims: Stress and anxiety during coronary angiography $(\mathrm{CA})$ can lead to serious complications such as cardiac dysrhythmias, coronary artery spasm, and coronary artery rupture. This study aimed to evaluating the effects of clinical simulation on stress, anxiety, and hemodynamic parameters among the candidates for CA.

Methods: This clinical trial was conducted on eighty candidates for CA. Participants were conveniently recruited from Tehran Heart Center, Tehran, Iran, and randomly allocated to a control and an intervention group through block randomization with a block size of 2. The Beck Anxiety Inventory, the Cohen Perceived Stress Scale, and a datasheet for hemodynamic parameters were used to assess participants' anxiety, stress, and hemodynamic parameters before and after the study intervention. Participants in the intervention group received routine care services and a clinical simulation intervention, while participants in the control group solely received routine care services. Data were analyzed using the chi-square, independent-sample $t$, and paired-sample $t$ tests.

Results: The mean scores of stress and anxiety and the mean values of systolic blood pressure (SBP), diastolic blood pressure (DBP), and heart rate $(\mathrm{HR})$ significantly decreased in the intervention group $(P<0.05)$, but did not significantly change in the control group $(P>0.05)$. The pretest posttest differences in the means of stress, anxiety, SBP, DBP, and HR in the intervention group were significantly greater than the control group $(P<0.05)$.

Conclusion: Clinical simulation is effective in significantly reducing stress, anxiety, and hemodynamic parameters among the candidates for CA. Therefore, clinical simulation is recommended before CA.

Keywords: Stress, Anxiety, Hemodynamic parameters, Clinical simulation
\end{abstract}

*Corresponding Author: Shahram Etemadifar, School of Nursing and Midwifery, Shahrekord University

of Medical Sciences,

Shahrekord, Iran.

Email: sh.etemadifar@yahoo. com

Received: 23 July 2020 Accepted: 9 November 2020 ePublished: 30 March 2021

\section{Introduction}

Coronary angiography (CA) is the gold standard for diagnosing coronary artery problems (1). It is also a low risk and effective procedure for managing some coronary artery problems (2). However, like other invasive procedures, CA is associated with high levels of stress and anxiety for patients (3). A study on 180 candidates for CA in Sari, Iran, reported that $55 \%$ of them had overt anxiety and $54 \%$ of them had covert anxiety (4).

Anxiety is an unpleasant state of strain caused by fear over disease, hospitalization, anesthesia, or surgery (5-7). Stress is also a psychological state in which individuals experience physical or psychological threat or instability. Stress severity largely depends on individuals' perceptions of potential or actual threats in a given situation so that an individual may experience little stress in a situation while another person may experience high levels of stress in that situation (8). Stress and anxiety can negatively affect physiological and hemodynamic parameters such as respiratory rate, heart rate (HR), myocardial oxygen consumption, plasma levels of epinephrine and norepinephrine, cardiac output, and blood pressure, and may put patients at risk for different health problems during CA $(5,6,9)$. Studies reported that anxiety is associated with increased risk of cardiac dysrhythmias, coronary artery spasm, and coronary artery rupture during CA $(10,11)$. Therefore, effective measures should be used to diagnose and manage CA-associated stress and anxiety in order to prevent potential risks (12). Vital signs assessment is one of the best methods for assessing stress and anxiety. Vital signs reflect physiological conditions in different conditions (13) and are valuable criteria for

(C) 2021 The Author(s); Published by Shahrekord University of Medical Sciences. This is an open-access article distributed under the terms of the Creative Commons Attribution License (https://creativecommons.org/licenses/by/4.0), which permits unrestricted use, distribution, and reproduction in any medium, provided the original work is properly cited. 
physiological assessment and clinical decision making (14). Studies showed that changes in vital signs had significant relationship with the levels of agitation $(5,16)$.

There are pharmacological and non-pharmacological therapies for stress and anxiety. Medication therapy by benzodiazepines and sedatives is the most common pharmacological therapy for stress and anxiety management. However, medication therapy has shortterm effects and is associated with different side effects. Therefore, non-pharmacological therapies have received great attention in recent years $(17,18)$. Simulation is one of the non-pharmacological therapies with potentials for stress and anxiety management. Simulation is used to imitate realities in clinical conditions for exercising processes, decision making, and critical thinking using techniques such as role playing and equipment such as videos or interactive mannequins (19). In simulation, real conditions are simulated to improve the transferability of the learned materials and solutions to real world and improve learners' understanding of the intended tasks. Simulation is associated with no risk of injury to learners.

Some previous studies reported the positive effects of simulation on learning outcomes among students. For instance, a study reported that using simulation-based teaching in clinical skills centers reduced learningrelated strain and promoted experiential and self-directed learning (20). A systematic review also showed the positive effects of using patient simulation mannequins on critical thinking skills and the ability to identify patient problems among nursing students (21). Simulation also helps effectively manage phobias $(22,23)$. A study into the effects of different teaching methods also showed that simulation produced better outcomes compared with conventional teaching methods (23). Moreover, a study reviewed different studies into the effects of clinical simulation and concluded that clinical simulation was associated with richer experiences compared with conventional teaching methods and helped learners face and correct their misconceptions (24).

Most previous studies into simulation evaluated its effects on students $(20,21)$. To the best of our knowledge, no study had yet evaluated the effects of simulation on stress and anxiety among candidates for CA. Therefore, the present study was conducted to narrow this gap. The aim of the study was to evaluate the effects of clinical simulation on stress, anxiety, and hemodynamic parameters among the candidates for CA.

\section{Methods \\ Design}

Using a single-blind two-group design, this clinical trial was conducted in 2016 in Tehran Heart Center, Tehran, Iran. Participants were eighty eligible candidates for CA. Eligibility criteria were an age of 20-70 years, affliction by class II or III cardiac problem according to the New York Heart Association classification, ability to read and write in Persian, no cognitive or anxiety disorder, no previous history of CA, no need for simultaneous CA and right heart catheterization (due to the likelihood of blood pressure fluctuations), no previous history of stressful diagnostic measures such as transesophageal echocardiography, no known valvular heart disease (due to its potential effects on hemodynamic status and anxiety), no in-depth knowledge about cardiac problems, no auditory verbal disorders, no family history of mental disorders, and consent for participation. Exclusion criteria were voluntary withdrawal from the study, need for emergency CA or hospitalization, and hospital discharge with personal consent before CA. Eligible CA candidates were conveniently recruited to the study and randomly allocated to a control $(n=40)$ and an intervention $(n=40)$ group through block randomization with a block size of 2 (12). Participants were blind to the study groups.

Sample size was calculated based on the results of three former studies $(13,14,25)$. Accordingly, with a confidence level of 0.95 and a $d$ of 2, sample size was determined to be 33 per group (Equation 1). Considering a potential attrition rate of $20 \%$, sample size was increased to forty per group.

$n=\frac{\left(z_{1-\alpha / 2}+z_{1-\beta}\right)^{2}\left(S_{1}^{2}+S_{2}^{2}\right)}{d^{2}}=\frac{(1.96+1.28)^{2}\left(2.5^{2}+2.5^{2}\right)}{2^{2}}=32.8 \mathrm{Eq}$.

\section{Instruments}

Data collection instruments were a demographic questionnaire, the Beck Anxiety Inventory, the Cohen Perceived Stress Scale, and a datasheet for hemodynamic parameters. The demographic questionnaire had items on age, gender, educational level, employment status, and insurance. The Beck Anxiety Inventory has 21 items scored on a $0-3$ scale with a possible total score of $0-63$. The score of this inventory is interpreted as follows: scores 0-21: low anxiety; scores 22-35: moderate anxiety; scores 36-63: severe anxiety with the need for assessment and treatment (26-28). A former study reported the acceptable validity and reliability of this inventory with a Cronbach's alpha of 0.92 (28). Other studies also reported this inventory as an appropriate anxiety measurement instrument in Iran (15,27,29-32).

The Cohen Perceived Stress Scale has ten items in three main subscales, namely unpredictability of life, uncontrollability of life, and overloadedness of life. Items are scored on a $0-4$ scale, resulting in a possible total score of $0-40$. Scores $0-10,11-20$, and 21-40 are interpreted as low, moderate, and severe perceived stress, respectively (33). Previous studies reported that the Cronbach's alpha of the scale was 0.78 (34), 0.85 (35), and 0.81 (36).

A datasheet was also used to document hemodynamic parameters, namely systolic blood pressure (SBP), diastolic blood pressure (DBP), and HR. Participants' blood pressure was measured using a sphygmomanometer. The sphygmomanometer was calibrated using another sphygmomanometer. The reliability of the sphygmomanometer was assessed through inter-rater reliability assessment, in which two assessors measured 
blood pressure with a five-minute interval without changing the place of the sphygmomanometer cuff. HR was also measured in a whole minute using an analogue watch.

Stress, anxiety, SBP, DBP, and HR in both groups were measured and documented at the time of hospital admission (i.e., one day before CA) and two hours before CA.

\section{Intervention}

Participants in the control group solely received care services routinely provided to all candidates for CA in the study setting, while their counterparts in the intervention group received both routine care services and clinical simulation. The clinical simulation intervention included five main components. The first component was a twentyminute nursing visit program, in which the researcher provided participants with educations about coronary artery disease, its etiology, its diagnostic procedures, and the benefits of CA and then, participants' probable misconceptions were assessed and corrected and their questions were answered. The second component was a twenty-minute video-based education about CA. The video was created in the $\mathrm{CA}$ and the recovery units of the study setting and included educations about patient entrance into the CA unit, patient preparation for CA, patient transfer from CA bed to stretcher, patient transfer from stretcher to bed in the recovery unit, and patient collaboration before, during, and after CA. The video was played for participants using a computer in the study setting. The third component was a CA unit tour. During the tour, participants were taken to the CA unit, where they were provided with educations about CA equipment, procedure, and process. The fourth component was mannequin-based education about patient preparation for $\mathrm{CA}$, puncture site preparation, and puncture site dressing after CA. In the fifth component, a trained hypothetical patient showed participants how to collaborate with CA team during the procedure and how to move from CA bed to stretcher. The simulation intervention was provided to participants in the intervention group in four- to sixperson small groups.

\section{Data analysis}

The SPSS software was used for data analysis. The independent-sample $t$ and the chi-square tests were used for between-group comparisons while the paired-sample $t$ test was used for within-group comparisons. The level of confidence was set at more than 0.95 .

\section{Results}

Eighty candidates for angiography in two forty-person groups participated in and completed this study. The mean of participants' age was $57 \pm 41$ years in total, 56.95 in the intervention group, and 57.87 in the control group. Most participants in the control and the intervention groups were male (73\% vs. $77 \%)$ and married (95\% vs.
95\%), had insurance (97\% vs. 97\%), and had belowdiploma education $(73 \%$ vs. $65 \%)$. At the beginning of the study, most participants reported high levels of stress (87\%) and anxiety (53\%). The results of the chi-square test revealed no statistically significant between-group differences respecting participants' gender, marital status, educational level, employment status, and insurance coverage $(P>0.05$; Table 1$)$.

The pretest mean score of stress was $22 \pm 1$ in the control group and $23 \pm 1$ in the intervention group and the posttest mean score of stress was $21 \pm 2$ in the control group and $13 \pm 4$ in the intervention group. Withingroup comparisons revealed no significant change in the mean score of stress in the control group $(P=0.680)$ and significant decrease in the mean score of stress in the intervention group $(P \leq 0.001)$. Between-group comparisons also indicated that although there was no significant difference between the groups respecting the pretest mean score of stress $(P=0.875)$, the posttest mean score of stress in the intervention group was significantly less than the control group $(P \leq 0.001$; Table 2$)$.

The pretest and the posttest mean scores of anxiety were respectively $33 \pm 3$ and $28 \pm 6.06$ in the control group and $32 \pm 5.02$ and $15 \pm 8.09$ in the intervention group. Withingroup comparisons showed that the mean score of anxiety did not significantly change in the control group $(P=0.725)$, while it significantly decreased in the intervention group $(P<0.001)$. Moreover, although there was no significant difference between the groups respecting the pretest mean score of anxiety $(P=0.521)$, the posttest mean score of anxiety in the intervention group was significantly less than the control group $(P<0.001$; Table 2$)$.

The pretest and the posttest mean values of SBP were respectively $133 \pm 6.02$ and $132 \pm 5.02$ in the control group and $135 \pm 7.02$ and $124 \pm 7.02$ in the intervention group. Within-group comparisons indicated no significant change in the mean value of SBP in the control group

Table 1. Between-group comparisons respecting participants' demographic characteristics

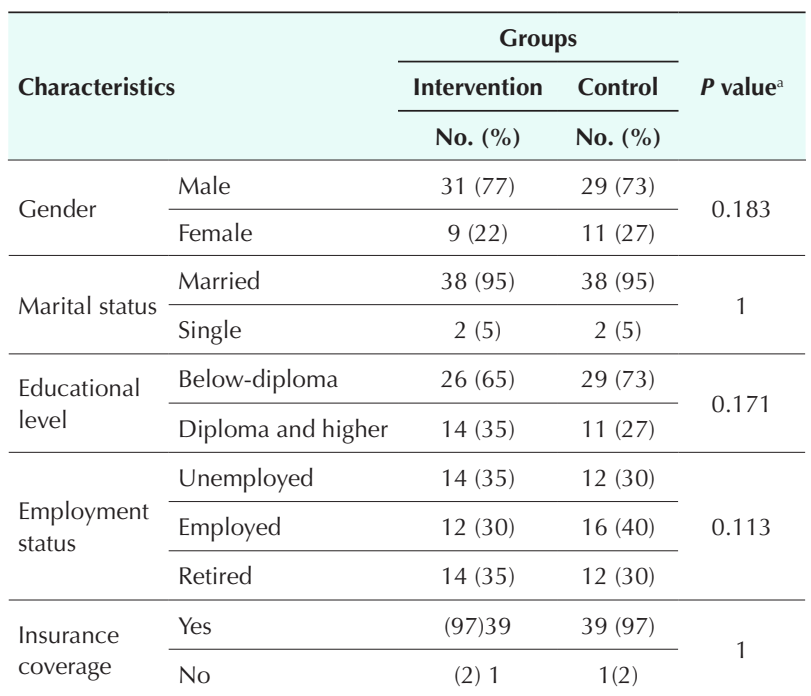

${ }^{a}$ Chi-square test. 
Table 2. Within- and between-group comparisons respecting the means scores of stress and anxiety and the mean values of SBP, DBP, and HR

\begin{tabular}{|c|c|c|c|c|c|}
\hline \multirow{3}{*}{ Variables } & \multirow{3}{*}{ Groups } & \multicolumn{2}{|c|}{ Time } & \multirow{3}{*}{$P$ value $^{\mathrm{a}}$} & \multirow{3}{*}{$\begin{array}{l}\text { Pretest posttest mean difference } \\
\text { Mean } \pm S D\end{array}$} \\
\hline & & Before & After & & \\
\hline & & Mean \pm SD & Mean \pm SD & & \\
\hline \multirow{3}{*}{ Stress } & Intervention & $23 \pm 1$ & $13 \pm 4$ & $<0.001$ & $9 \pm 1$ \\
\hline & Control & $22 \pm 1$ & $21 \pm 2$ & 0.680 & $2 \pm 1$ \\
\hline & $P$ value ${ }^{b}$ & 0.875 & 0.001 & - & - \\
\hline \multirow{3}{*}{ Anxiety } & Intervention & $32 \pm 5.02$ & $15 \pm 8.09$ & 0.001 & $17 \pm 8$ \\
\hline & Control & $33 \pm 3.02$ & $28 \pm 6.06$ & 0.725 & $5 \pm 4$ \\
\hline & $P$ value ${ }^{b}$ & 0.521 & 0.001 & - & - \\
\hline \multirow{3}{*}{ SBP } & Intervention & $135 \pm 7.02$ & $124 \pm 7.02$ & $<0.002$ & $6 \pm 4$ \\
\hline & Control & $133 \pm 6.02$ & $132 \pm 5.02$ & $<0.865$ & $1 \pm 3$ \\
\hline & $P$ value $^{\mathrm{b}}$ & 0.575 & $<0.003$ & - & - \\
\hline \multirow{3}{*}{ DBP } & Intervention & $82 \pm 4.01$ & $76 \pm 3.02$ & $<0.003$ & $6 \pm 4$ \\
\hline & Control & $81 \pm 5.02$ & $80 \pm 4.01$ & $<0.665$ & $4 \pm 1$ \\
\hline & $P$ value $^{b}$ & $<0.875$ & $<0.005$ & - & - \\
\hline \multirow{3}{*}{$H R$} & Intervention & $80 \pm 4.02$ & $71 \pm 3.02$ & $<0.002$ & $8 \pm 4$ \\
\hline & Control & $79 \pm 3.02$ & $78 \pm 3.02$ & $<0.865$ & $1 \pm 4$ \\
\hline & $P$ value ${ }^{b}$ & $<0.865$ & $<0.001$ & - & - \\
\hline
\end{tabular}

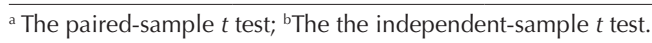

$(P=0.865)$ and a significant decrease in the mean value of SBP in the intervention group $(P \leq 0.002)$. Betweengroup comparisons also showed that while there was no significant difference between the groups respecting the pretest mean value of SBP $(P=0.003)$, the posttest mean value of SBP in the intervention group was significantly less than the control group $(P<0.001$; Table 2$)$.

The pretest and the posttest mean values of DBP were respectively $81 \pm 5.02$ and $80 \pm 4.01$ in the control group and $82 \pm 4.01$ and $76 \pm 3.02$ in the intervention group. Although no significant change was observed in the mean value of DBP in the control group $(P=0.665)$, the mean value of DBP significantly decreased in the intervention group $(P<0.003)$. Moreover, there was no between-group difference respecting the pretest mean value of DBP $(P=0.875)$, while the posttest mean value of DBP in the intervention group was significantly less than the control group $(P<0.005)$.

The pretest and the posttest mean values of $\mathrm{HR}$ were $79 \pm 3.02$ and $78 \pm 3.02$ in the control group and $80 \pm 4.02$ and $71 \pm 3.02$ in the intervention group. Although the within-group change in the mean value of HR was not statistically significant in the control group $(P=0.865)$, the mean value of $\mathrm{HR}$ significantly decreased in the intervention group $(P<0.001)$. Moreover, between-group difference respecting the pretest mean value of HR was not significant $(P=0.865)$, while the posttest mean value of HR in the intervention group was significantly less than the control group $(P<0.001$; Table 2$)$.

Between-group comparisons respecting the pretest posttest mean differences also showed that pretest posttest changes in the mean scores of stress and anxiety and the mean values of SBP, DBP, and HR in the intervention group were significantly greater than the control group $(P<0.05$; Table 2).

\section{Discussion}

This study evaluated the effects of clinical simulation on stress, anxiety, and hemodynamic parameters among the candidates for CA. Findings indicated the significant positive effects of clinical simulation on stress, anxiety, and hemodynamic parameters.

Our findings showed that clinical simulation significantly reduced stress among the candidates for CA. In agreement with this finding, two former studies showed the effectiveness of orientation programs in significantly reducing stress among patients undergoing CA (26,37-43). Another study showed that mind simulation significantly reduced stress and increased cognitive flexibility among adults with stuttering disorder (41). Simulation has significant roles in ensuring patient safety and manipulating or predicting behaviors (37). It helps create an artificial environment for safely exercising activities without causing serious injuries (39). Before attending real clinical environment, most patients experience some levels of anxiety and stress mostly due to having limited knowledge. Accordingly, they prefer to receive education about the environment and even have a brief visit of it. Simulation provides patients with the opportunity to safely visit clinical settings and receive education about accurate behaviors in different situations and thereby, reduces their stress and anxiety, improves their ability to perform tasks, increases their self-confidence, strengthens their relationships with healthcare providers, and gives them a sense of worthiness (40).

Study findings also showed no significant between- 
group difference respecting the pretest mean score of anxiety, denoting the homogeneity of the groups in terms of baseline anxiety level. Several earlier studies also reported the same finding (42-44). Moreover, findings showed that clinical simulation significantly reduced anxiety among the candidates for CA. A former study also showed the effectiveness of a CA orientation tour in significantly reducing anxiety among patients (45). Another study reported that simulation-based learning can significantly reduce anxiety and stress among patients and learners (46). Two other studies also reported the positive effects of simulation on anxiety among patients $(26,44)$. Almost all patients experience some levels of stress and anxiety when they attend healthcare settings to receive healthcare services due to unfamiliarity with healthcare environment, separation from family members, and imaginations about diagnostic and therapeutic procedures and their results (47). They attribute their stress and anxiety to factors such as fear over the unknown, potential procedural threats, necessity for surgery, CA complications, and uncertainties over the future. Fear over the unknown is mainly due to the lack of knowledge (26). Stress, anxiety, and their contributing factors can endanger patient life during CA and cause them serious complications such as cardiac dysrhythmias, coronary artery spasm, or even coronary artery rupture $(11,26)$. Therefore, careful stress and anxiety assessment is needed before CA in order to identify patients with high levels of stress and anxiety, take appropriate measures for managing their stress and anxiety, and improve the accuracy and the outcomes of CA (12). Unlike our findings, two previous studies reported that orientation programs had no significant effects on procedural anxiety among patients $(48,49)$. This contradiction is attributable to the differences among studies respecting their interventions, anxiety measurement protocols, and baseline anxiety levels.

We also found that clinical simulation significantly reduced SBP, DBP, and HR among the candidates for CA. A previous study also reported the significant positive effects of an orientation program on hemodynamic parameters (26). Another study revealed that anxiety reduction training had significant positive effects on vital signs among candidates for elective surgeries (50).

Like other studies, this study had some limitations. For instance, participants might have acquired information about CA from media or from patients with previous CA experience. Such information might have affected their stress and anxiety.

\section{Conclusion}

This study concludes that clinical simulation is effective in significantly reducing stress, anxiety, SBP, DBP, and HR among the candidates for CA. Therefore, simulationbased interventions are recommended for CA candidates.

\section{Conflict of Interests}

None declared.
What does this paper contribute to the wider global clinical community?

- Clinical simulation is effective in significantly reducing stress, anxiety, and hemodynamic parameters among the candidates for coronary angiography.

- Clinical simulation can be used to improve patient satisfaction.

\section{Ethical Approval}

The Ethics Committee of Shahrekord University of Medical Sciences, Shahrekord, Iran, approved this study (code: IR.SKUMS.REC.1395.294). Moreover, the study was registered in the Iranian Registry of Clinical Trials (code: IRCT2017061734584N1).

\section{Acknowledgement}

We would like to thank the head nurses and the staff of the CA and the coronary care units of the study setting, the patients who agreed to participate in the study, and the Research Administration of Shahrekord University of Medical Sciences, Shahrekord, Iran.

\section{References}

1. Wilson RF. Coronary angiography. In: Coronary Artery Disease. Springer; 2015. p. 69-144.

2. Bunevicius A, Staniute M, Brozaitiene J, Pop VJ, Neverauskas J, Bunevicius R. Screening for anxiety disorders in patients with coronary artery disease. Health Qual Life Outcomes. 2013;11:37. doi: 10.1186/1477-7525-11-37.

3. Thompson DR, Chair SY, Chan SW, Astin F, Davidson PM, Ski CF. Motivational interviewing: a useful approach to improving cardiovascular health? J Clin Nurs. 2011;20(9-10):1236-44. doi: 10.1111/j.13652702.2010.03558.x.

4. Tahmasbi H, Mahmoodi G, Mokhberi V, Hassani $\mathrm{S}$, Akbarzadeh $\mathrm{H}$, Rahnamai $\mathrm{N}$. The impact of aromatherapy on the anxiety of patients experiencing coronary angiography. Zahedan J Res Med Sci. 2012;14(3):51-5. [Persian].

5. Majidi S. Recitation effect of holy Quran on anxiety of patients before undergoing coronary artery angiography. J Guilan Univ Med Sci. 2004;13(49):61-7. [Persian].

6. Beddoes L, Botti M, Duke MM. Patients' experiences of cardiology procedures using minimal conscious sedation. Heart Lung. 2008;37(3):196-204. doi: 10.1016/j.hrtlng.2007.05.012.

7. Torabi M, Salavati M, Ghahri Sarabi A, Pouresmaeil Z. Effects of reflexology foot massage and Benson relaxation on anxiety and physiological parameters of patients candidate for angiography. Avicenna J Nurs Midwifery Care. 2012;20(1):63-71. [Persian].

8. Moghadam M, Rashidzadeh S, Shamsalizadeh $N$, Fallahi B. The impact of stress management training on the copping style and perceived stress in medical students. Sci J Kurdistan Univ Med Sci. 2014;19(3):5260. doi: 10.22102/19.3.6. [Persian].

9. Turton MB, Deegan T, Coulshed N. Plasma catecholamine levels and cardiac rhythm before and after cardiac catheterisation. $\mathrm{Br}$ Heart J. 1977;39(12):1307-11. doi: 10.1136/hrt.39.12.1307. 
10. Hanifi $N$, Bahraminejad $N$, Idea Dadgaran SA, Ahmadi F, Khani M, Haghdoost Oskouie SF. Effect of orientation program on hemodynamic variables of patients undergoing heart catheterization. Hayat. 2011;17(3):38-48. [Persian].

11. Khayyam-Nekouei Z, Yousefy A, Manshaee G, Nikneshan S. Comparing anxiety in cardiac patients candidate for angiography with normal population. ARYA Atheroscler. 2011;7(3):93-6.

12. Mohammadi N, Tizhoosh $M$, Seyedoshohadaei $M$, Haghani $\mathrm{H}$. Face-to-face education vs. group education on knowledge and anxiety of patients undergoing coronary angiography. Hayat. 2012;18(3):44-53. [Persian].

13. Castledine G. The importance of measuring and recording vital signs correctly. Br J Nurs. 2006;15(5):285. doi: 10.12968/bjon.2006.15.5.20645.

14. Burykin A, Peck T, Krejci V, Vannucci A, Kangrga I, Buchman TG. Toward optimal display of physiologic status in critical care: I. Recreating bedside displays from archived physiologic data. J Crit Care. 2011;26(1):105. e1-105.e9. doi: 10.1016/j.jcrc.2010.06.013.

15. Chase JG, Starfinger C, Lam Z, Agogue F, Shaw GM. Quantifying agitation in sedated ICU patients using heart rate and blood pressure. Physiol Meas. 2004;25(4):1037-51. doi: 10.1088/09673334/25/4/020.

16. Janz BA, Clifford GD, Mietus JE, Mark RG. Multivariable analysis of sedation, activity, and agitation in critically ill patients using the Riker scale ECG, blood pressure, and respiratory rate. In: Computers in Cardiology. Lyon, France: IEEE; 2005. p. 735-8. doi: 10.1109/ cic.2005.1588209.

17. Mahdavi A, Heidari Gorji MA, Heidari Gorji AM, Yazdani J, Didehdar Ardebil M. Implementing Benson's relaxation training in hemodialysis patients: changes in perceived stress, anxiety, and depression. $\mathrm{N}$ Am J Med Sci. 2013;5(9):536-40. doi: 10.4103/19472714.118917.

18. Zargarzadeh M, Memarian R, Rafiee A. The effect of progressive muscle relaxation program on chronic low back pain and living daily activities in patients with intervertebral disc: a randomized clinical trial. J Shahrekord Univ Med Sci. 2015;16(6):101-10. [Persian].

19. Gaberson KB, Oermann MH, Shellenbarger T. Clinical Teaching Strategies in Nursing. Springer Publishing Company; 2014.

20. Freeth D, Fry $H$. Nursing students' and tutors' perceptions of learning and teaching in a clinical skills centre. Nurse Educ Today. 2005;25(4):272-82. doi: 10.1016/j.nedt.2005.01.007.

21. Lapkin S, Levett-Jones $T$, Bellchambers $H$, Fernandez R. Effectiveness of patient simulation manikins in teaching clinical reasoning skills to undergraduate nursing students: a systematic review. Clin Simul Nurs. 2010;6(6):e207-e22. doi: 10.1016/j.ecns.2010.05.005.

22. Gutierrez M, Vexo F, Thalmann D. Stepping into Virtual Reality. Springer Science \& Business Media; 2008.

23. Salas E, Tannenbaum SI, Kraiger K, Smith-Jentsch KA. The science of training and development in organizations: what matters in practice. Psychol Sci Public Interest. 2012;13(2):74-101. doi: 10.1177/1529100612436661.

24. Strangman N, Hall T. Virtual Reality/Simulations. Wakefield, MA: National Center on Accessing the
General Curriculum; 2006.

25. Eom MR, Kim HS, Kim EK, Seong K. [Effects of teaching method using standardized patients on nursing competence in subcutaneous injection, self-directed learning readiness, and problem solving ability]. J Korean Acad Nurs. 2010;40(2):151-60. doi: 10.4040/ jkan.2010.40.2.151.

26. Hanifi N, Bahraminejad N, Mirzaei Khalil Abadi T, Ahmadi F, Khani M, Taran L. The effect of orientation program on stress, anxiety and depression of patients undergoing coronary angiography. Iran J Nurs Res. 2012;7(25):1-8. [Persian].

27. Beck AT, Epstein N, Brown G, Steer RA. An inventory for measuring clinical anxiety: psychometric properties. J Consult Clin Psychol. 1988;56(6):893-7. doi: 10.1037//0022-006x.56.6.893.

28. Kaviani H, Ahmadi Abhari A, Dehghan M, Mansournia MA, Khorramshahi $M$, Ghadirzadeh $M R$, et al. Prevalence of anxiety disorders in Tehran city. Iran J Psychiatry Clin Psychol. 2003;8(3):4-11. [Persian].

29. Beck AT, Steer RA. Relationship between the beck anxiety inventory and the Hamilton anxiety rating scale with anxious outpatients. J Anxiety Disord. 1991;5(3):213-23. doi: 10.1016/0887-6185(91)90002b.

30. Osman A, Kopper BA, Barrios FX, Osman JR, Wade T. The Beck Anxiety Inventory: reexamination of factor structure and psychometric properties. J Clin Psychol. 1997;53(1):7-14. doi: 10.1002/(sici)10974679(199701)53:1 < 7::aid-jclp2 > 3.0.co;2-s.

31. Osman A, Hoffman J, Barrios FX, Kopper BA, Breitenstein JL, Hahn SK. Factor structure, reliability, and validity of the Beck Anxiety Inventory in adolescent psychiatric inpatients. J Clin Psychol. 2002;58(4):44356. doi: 10.1002/jclp.1154.

32. Kaviani H, Mousavi AS. Psychometric properties of the Persian version of Beck Anxiety Inventory (BAI). Tehran Univ Med J. 2008;66(2):136-40. [Persian].

33. Cohen S. Perceived stress in a probability sample of the United States. In: Spacapan S, Oskamp S, eds. The Social Psychology of Health. Sage Publications Inc; 1988. p. 31-67.

34. Ezzati A, Jiang J, Katz MJ, Sliwinski MJ, Zimmerman ME, Lipton RB. Validation of the Perceived Stress Scale in a community sample of older adults. Int J Geriatr Psychiatry. 2014;29(6):645-52. doi: 10.1002/gps.4049.

35. Cohen S, Janicki-Deverts D. Who's stressed? distributions of psychological stress in the United States in probability samples from 1983, 2006, and 20091. J Appl Soc Psychol. 2012;42(6):1320-34. doi: 10.1111/j.1559-1816.2012.00900.x.

36. AraghianMojarad F, Mahmoodi Shan Gholam R, DANESH A, Roshandel GR. Relationship between Perceived Stress and Coping Strategies of Relatives of Patients Hospitilzed in Cardiac Care. Unit journal of nursing education. 2020;9(1):19-28.

37. Boulet JR, Murray DJ. Simulation-based assessment in anesthesiology: requirements for practical implementation. Anesthesiology. 2010;112(4):104152. doi: 10.1097/ALN.0b013e3181cea265.

38. Grant MM, Davis KH. Simulation-Based Learning in Medical Laboratory Education. Canadian Society for Medical Laboratory Science (CSMLS); 2007. p. 5-14.

39. Murray C, Grant MJ, Howarth ML, Leigh J. The use of simulation as a teaching and learning approach 
to support practice learning. Nurse Educ Pract. 2008;8(1):5-8. doi: 10.1016/j.nepr.2007.08.001.

40. McCallum J. The debate in favour of using simulation education in pre-registration adult nursing. Nurse Educ Today. 2007;27(8):825-31. doi: 10.1016/j. nedt.2006.10.014.

41. Taghizadeh ME, Yarollahi NA, Bahrami Z. The effectiveness of the simulation mind model on reducing stress and increasing cognitive flexibility in adult with stuttering disorder. Shenakht Journal of Psychology and Psychiatry. 2018;5(2):67-80. doi: 10.29252/ shenakht.5.2.67. [Persian].

42. Nikbakht Nasrabadi AR, Bakhshayeshi O, Parsayekta Z, Hoseyni M, Taghavi T, Rezvani H. The effectiveness of implementing nursing consultation on the anxiety of patients undergoing Gl endoscopy. Iran Journal of Nursing. 2012;25(79):54-62. [Persian].

43. Varaei S, Keshavarz S, Nikbakht Nasrabadi AR, Shamsizadeh $M$, Kazemnejad $A$. The effect of orientation tour with angiography procedure on anxiety and satisfaction of patients undergoing coronary angiography. Iranian Journal of Psychiatric Nursing. 2013;1(2):1-10. [Persian ].

44. Varaei S, Shamsizadeh M, Cheraghi MA, Talebi M, Dehghani A, Abbasi A. Effects of a peer education on cardiac self-efficacy and readmissions in patients undergoing coronary artery bypass graft surgery: a randomized-controlled trial. Nurs Crit Care. 2017;22(1):19-28. doi: 10.1111/nicc.12118.

45. Kolahi Z, Zandi M, Esmaeili R, KhabazKhoob M. Effect of angiography room orientation tour on anxiety of patients awaiting cerebrovascular angiography. Advances in Nursing \& Midwifery. 2020;29(2):7-11.

46. Sajadi SA, Farsi Z. Simulation-based education. Nama. 2015;3(2):20-30. [Persian].

47. Jamshidi N, Abaszade A, Najafi-Kaliani M. Stress, anxiety and depression of patients before coronary angiography. Zahedan J Res Med Sci. 2012;13(Suppl 1):e95207. [Persian]

48. Taylor-Piliae RE, Chair SY. The effect of nursing interventions utilizing music therapy or sensory information on Chinese patients' anxiety prior to cardiac catheterization: a pilot study. Eur J Cardiovasc Nurs. 2002;1(3):203-11. doi: 10.1016/s14745151(02)00037-3.

49. Talaei A, Toofani H, Hojjat K, Jami Z. The effect of orientation program with operation room on anxiety of patient candidate for tubectomy. J Fundament Health. 2004;6(21-22):57-61.

50. Bahrami N, Soleimani MA, Sharifnia H, Shaigan $H$, Sheikhi MR, Mohammad-Rezaei Z. Effects of anxiety reduction training on physiological indices and serum cortisol levels before elective surgery. Iran J Nurs Midwifery Res. 2013;18(5):416-20. 